\title{
Genetic Complementation Studies of Human Pin1 in Azotobacter vinelandii Revealed that it Requires Amino Terminus of the NifM to Deliver PPIase Effect to the Fe-protein of Nitrogenase
}

\author{
Kumaraguru Raja, Lakshmi Pulakat, and Nara Gavini \\ Department of Biological Sciences, Mississippi State University \\ Mississippi State, MS 39762, USA
}

\begin{abstract}
The NifM is a peptidyl prolyl cis-trans isomerase and is required for the maturation and activation of the Fe protein of Nitrogenase. Since the carboxyl terminus of NifM is similar to the Human Pin1, we expressed the Human Pin1 in A. vinelandii BG98, a nifM mutant strain containing a kanamycin insertion and found that it could not complement the function of nifM. It was hypothesized that the amino terminus of the NifM might be required for the Pin1 to bind to NifH similar to requirement of the WW domain for the binding to $\mathrm{pSer} / \mathrm{Thr}$-Pro of $\mathrm{Cdc} 25 \mathrm{C}$. Therefore we expressed a NifM amino-terminal and Pin1 fusion protein chimera in A. vinelandii BG98 and this chimera was able to complement the function of NifM. This observation indicated that the amino-terminal of NifM is responsible for the specificity for NifH similar to the specificity of WW domain of Pin1 for pSer/ThrPro while the carboxyl terminal domain functions as peptidyl prolyl cis/trans isomerase.
\end{abstract}

Key words: Azotobacter vinelandii, Peptidyl prolyl cis/trans isomerase, Human Pin1,NifM-Pin1 chimara, Genetic complementation.

\section{INTRODUCTION}

Nitrogenase, the enzyme responsible for conversion of atmospheric nitrogen to ammonia, consists of two separable protein components the $\mathrm{Fe}$ protein and the MoFe protein, both of which are extremely oxygen-sensitive [1-3]. The Fe protein is a $64 \mathrm{kDa}$ homodimer encoded by nifH $[4,5]$. The Fe protein has a single $4 \mathrm{Fe}: 4 \mathrm{~S}$ cluster covalently bridging each subunit and can bind up to two MgATP and/or MgADP molecules per dimer, and is essential for coupling nucleotide hydrolysis to electron transfer [5]. The products of the nif structural genes nifHDK are not catalytically competent and need to be further processed to active forms by the action of a number of nif accessory genes [6]. The nif $M$ gene product is required for the accumulation of the active $\mathrm{Fe}$ protein in $K$. pneumoniae [7], as well as in foreign host $E$. coli $[8,9]$ and for dimerization in S. cerevisiae [10]. Mutation of the nifM gene results in the slower accumulation of $\mathrm{Fe}$ protein, indicating that NifM is involved in the correct folding of the Fe protein [11]. In $A$. vinelandii, the nifU and nifS together with the nifM gene product are required for full stability and activation of the Fe protein $[12,13]$.
NifM is a peptidyl prolyl cis/trans isomerase (PPIase) required for the activation and maturation of the Fe protein of nitrogenase [14]. PPIases are a class of enzymes that accelerate protein folding by catalyzing the cis-trans isomerization of proline imidic peptide bonds in oligopeptides $[15,16]$.

Pin1 is a phosphorylation dependent PPIase that isomerizes proline residues preceded by a phosphorylated serine or threonine in substrates with up to 1300-fold selectivity compared with nonphosphrylated peptides [17]. The Pin1 is a highly conserved enzyme and has been shown to be involved in various diverse biological processes and pathological conditions like cancer and Alzheimer's disease [18]. The Pin1- type parvulin proteins are comprised of two domains an amino terminal WWdomain and a carboxyl terminal PPIase domain, each of which is highly conserved between the species [19]. The WW domain is thought to be required for substrate specificity, specifically the interaction with phosphoserine or phosphothreonine containing proteins [20]. It has been shown that the WW domain functions as a phosphoserine or phosphothreoninebinding module [21]. The WW domain is a protein-protein interaction module found in many other unrelated proteins and characterized by the

Corresponding Author: Nara Gavini, Department of Biological Sciences, Mississippi State University, Mississippi State, MS 39762, USA 
conservation of two specific tryptophan residues spaced by 22 amino acid residues [20]. E. coli parvulin contains no WW domain, but is still an active PPIase [22].

The carboxyl terminus of NifM protein shares significant homology with the PPIase family of proteins (14). It has been shown by protease coupled assay that NifM has a PPIase activity comparable to that of PpiC of E.coli (14). In A. vinelandii, the NifM protein is 292 amino acid residues long (12) and has a 96 amino acid residue PPIase domain that spans from amino acid 148 to 243. NifM is required for the activity of all the three nitrogenases (23). Here, we report that the aminoterminal of NifM may be responsible for the specificity for $\mathrm{NifH}$ similar to the specificity of the WW domain of Pin1 for pSer/Thr-Pro.

Table 1: Bacterial strains and plasmids used in this study.

\begin{tabular}{|c|c|c|}
\hline Strain/ Plasmid & Relevant characteristics and description & Source/reference \\
\hline E. coli $\mathrm{TG} 1$ & K-12 $\Delta$ (lac-pro) supE thi hsd-5/F'traD36 rroA $^{+} B^{+}$lacI $^{q}$ lacZ $\Delta M 15$ & $\begin{array}{l}\text { AmershamLife } \\
\text { Sciences, Inc. }\end{array}$ \\
\hline A. vinelandii $\mathrm{OP}$ & Wild type, Nif+, soil bacterium & 26 \\
\hline A. vinelandii BG98 & $\begin{array}{l}\text { Nif-; defined deletion in the nifM gene. } 309 \text { bp of SmaI fragment from nifM is } \\
\text { replaced by kanamycin cassette }\end{array}$ & 14 \\
\hline A. vinelandii $\mathrm{BG} 3302$ & $\begin{array}{l}\text { Nif -; chloramphenicol-resistant, generated by transformation with plasmid } \\
\text { pBG3302 }\end{array}$ & This work \\
\hline A. vinelandii $\mathrm{BG} 3305$ & $\begin{array}{l}\text { Nif +; chloramphenicol-resistant, generated by transformation with plasmid } \\
\text { pBG3305 }\end{array}$ & This work \\
\hline $\mathrm{pCR} \circledast 2.1 \mathrm{TOPO}$ & $\begin{array}{l}\text { AmpR, KanR, 3908bp used for direct cloning of PCR products, lacZ } \alpha \text { fragment, } \\
\text { MCS, M13 }\end{array}$ & $\begin{array}{l}\text { Invitrogen, } \\
\text { Inc.,Carlsbad,CA }\end{array}$ \\
\hline pBG1380 & Derivative of pBHR1 Promoter nifH-7his/pBHR1 PstI-del & 14 \\
\hline pBG3301 & $\begin{array}{l}\text { Derivative of pCR® } 2.1 \text { TOPO in which } 492 \text { bp DNA fragment of Pin } 1 \text { was cloned } \\
\text { directly after PCR using appropriate oligonucleotide primers designed with flanking } \\
\text { BamH1 sites }\end{array}$ & This work \\
\hline pBG3304 & $\begin{array}{l}\text { Derivative of pCR } 2.1 \text { TOPO in which from } 378 \text { bp DNA fragment of nifM was } \\
\text { cloned directly after PCR using appropritate oligonucleotide primers designed with } \\
\text { flanking EcoRV sites }\end{array}$ & This work \\
\hline pBG3302 & $\begin{array}{l}\text { Derivative of pBG1380 in which } 492 \text { bp DNA fragment of pin } 1 \text { was cloned in pBG } \\
1380\end{array}$ & This work \\
\hline
\end{tabular}




\section{MATERIALS AND METHODS}

Bacterial strains, plasmids and growth conditions: The bacterial strains and plasmids used in this study are listed in Table 1. A. vinelandii strains were grown at $30^{\circ} \mathrm{C}$ in modified Burks's nitrogen free $\left(\mathrm{BN}^{-}\right)$medium [24] whenever nitrogen deficient medium was needed, and when nitrogen was required $\mathrm{BN}^{-}$medium supplemented with $400 \mu \mathrm{g} / \mathrm{ml}$ of ammonium acetate was used. The $E$. coli strains were grown at $37^{\circ} \mathrm{C}$ in $2 \mathrm{YT}$ broth [25]. Chloramphenicol and ampicillin were used at a final concentration of $34 \mu \mathrm{g} / \mathrm{ml}$ and $50 \mu \mathrm{g} / \mathrm{ml}$ respectively, wherever the selection was made.

General molecular biology techniques: Restriction enzymes were purchased from Promega, Inc. (Madison, WI). DNA sub-cloning, plasmid DNA isolations, restriction enzyme digestions, agarose gel electrophoresis, ligations and E. coli transformations were carried out as described in the laboratory manual or according to the manufacturer's protocol
[25]. Oligonucleotides used for PCR amplification were purchased from GIBCO BRL Life Technologies, Inc (Gaithersburg, MD).

Transformation of $A$. vinelandii and growth characteristics: The transformations of $A$. vinelandii strains were carried out as described previously [24, 26]. Since the plasmids pBG3302, pBG3305 and pBG3311 are derivatives of pBHR1 a broad-hostrange vector [27], they are capable of replicating at medium copy numbers in $A$. vinelandii. These plasmids have chloramphenicol resistance marker and express the cloned genes under the control of nif $\mathrm{H}$ promoter. Therefore, these plasmids could be used to express proteins in A. vinelandii after transformation and growing under nitrogen limiting conditions. The transformants were selected on $\mathrm{BN}^{+}$ medium containing $68 \mu \mathrm{g} / \mathrm{ml}$ chloramphenicol. Growth characteristics of the transformants were determined by growth curve analysis as described previously $[23,26]$.

\section{Error! \\ Pin1 \\ NifM \\ MASERLADGD SRYYLLKVAHEQFGCAP GELSEDQLQQADRI I GRQRH IED \\ Pin1 \\ NifM \\ AVLRSPDAIGVVIPP SQLEEAWAHIASRYESPEALQQALDAQALDAAGMR \\ Pin1 \\ NifM

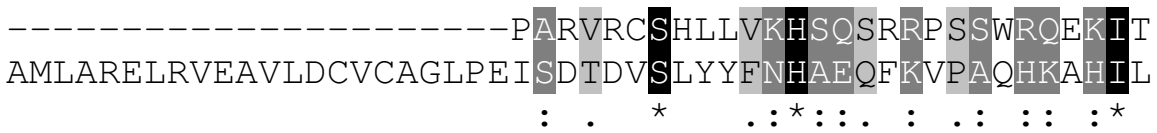 \\ Pin 1 \\ NifM

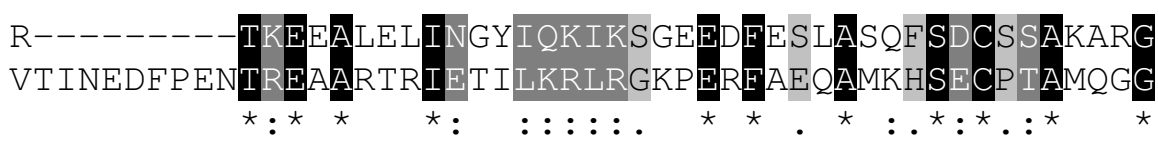 \\ Pin1 \\ NifM

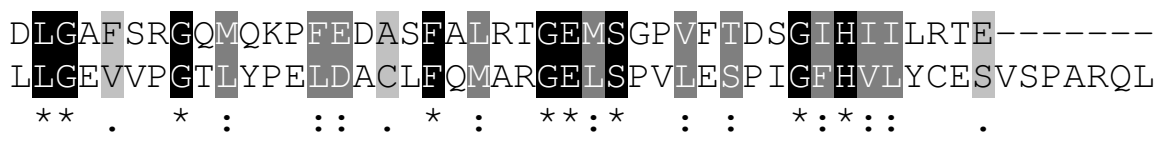 \\ $\operatorname{Pin} 1$ \\ $\mathrm{NifM}$ \\ TLEEILPRLRDRLQLRQRKAYQRKWLVCLLQQNATLENLAHG \\ Fig. 1: Amino acid sequence comparison of the peptidyl prolyl cis-trans isomerase domain of the Human Pin1 protein and NifM protein from A. vinelandii. The amino acid sequences were obtained from GenBank (http://www.ncbi.nlm.nih.gov). The data was uploaded to San Diego Supercomputer Center (http://workbench.sdsc.edu) for further analyses. The sequences were aligned using the Clustal W program (29). Maximum gap and mismatch penalties were opted for when the comparison was performed. A number of residues labeled by asterisks/black shades were fullyconserved. Some labeled by semicolons/dark gray shades were highly conserved; the ones labeled by periods/light gray shades were weakly conserved. The other residues without any labels were not conserved. The peptidyl prolyl cis-trans isomerase domain of the Human Pin1 is similar to the peptidyl prolyl cis-trans isomerase domain of NifM and maps to the carboxyl terminus of NifM.}



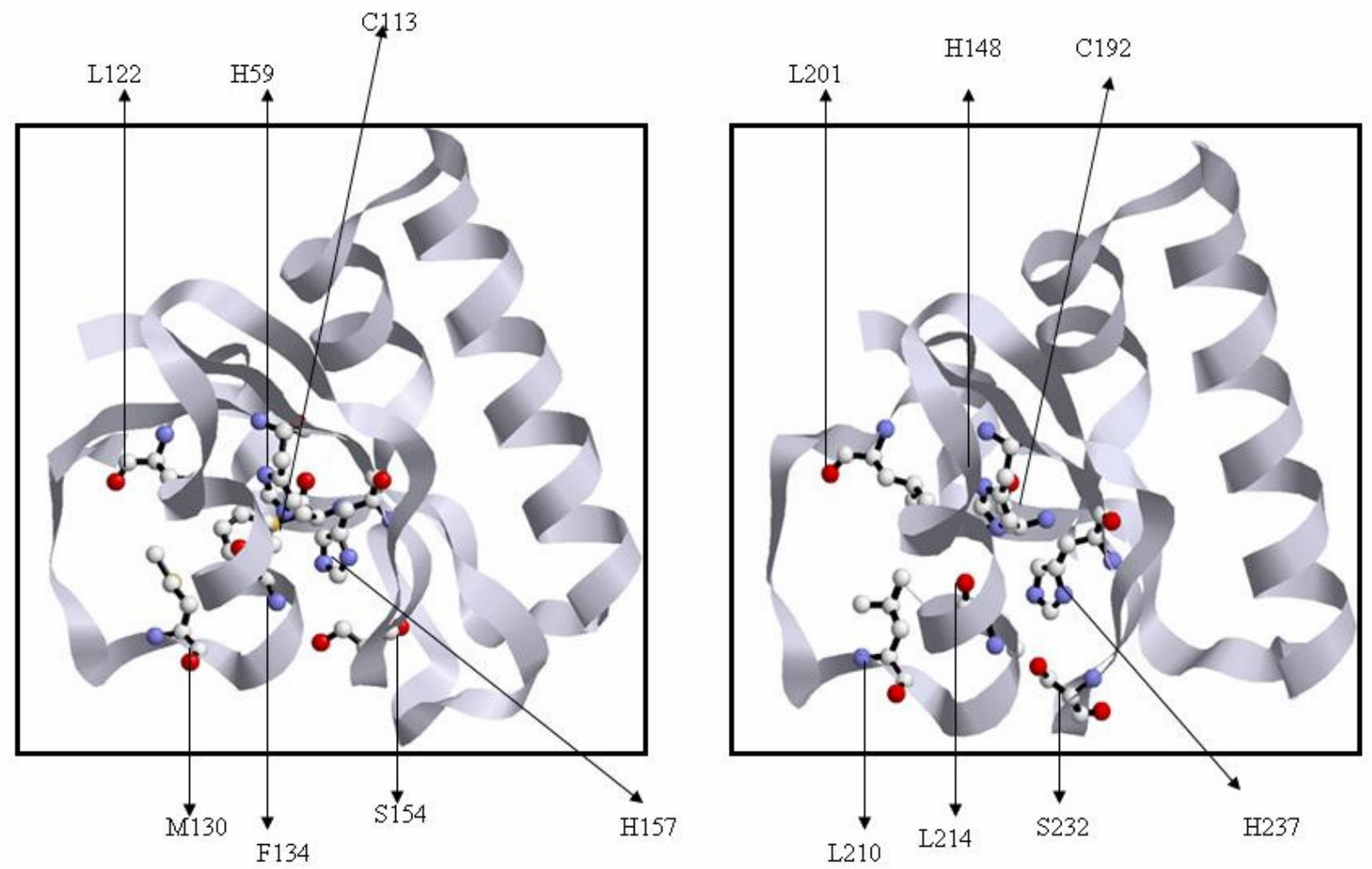

Fig. 2: Comparison of the structure of the PPIase domain of Pin1 to that of the PPIase domain of NifM. The model for NifM PPIase domain was built using the PPIase domain of the crystal structure of Human Pin1( pdb code: 1PIN1) as template. Homology modeling was performed using the program SwissPdb Viewer (30). The hydrophobic substate-binding pocket is conserved in both the Pin1 and the NifM, the hydrophobic pocket is made up of residues L122, M130 and F134 in Pin1, and it consists of residues L201, L210 and L214 in the NifM. While the residues H59, C113, H157 and S154 interact with the substrate in Pin1 the corresponding residues conserved in the NifM are H148, C192, H237 and S232. The residues, which interact with the substrate, are conserved in both the NifM as well as Pin1.

\section{RESULTS AND DISCUSSION}

Human Pin1 Protein does not complement the NifM function in $A$. vinelandii: Sequence comparison of Human Pin1 and the NifM of $A$. vinelandii revealed that the peptidyl prolyl cis-trans isomerase domain of the Human Pin1 is similar to the peptidyl prolyl cis-trans isomerase domain of NifM and this sequence similarity is confined to the carboxyl terminus of NifM (Fig.1). The hydrophobic substrate-binding pocket is conserved in both the Pin1 and the NifM, while the hydrophobic pocket is made up of residues L122, M130 and F134 in Pin1 [28] it is made up L201, L210 and L214 in the NifM (Fig.2). The residues, which interact with the substrate, are conserved in both the NifM as well as Pin1.While the residues H59, C113, H157 and S154 interact with the substrate in Pin1 [28] the corresponding residues conserved in the NifM are H148, C192, H237 and S232 (Fig.2). Based on these similarities it is predicted that the catalytic domain of the Pin1 as well the NifM function in a similar manner. A 492bp DNA fragment encoding the peptidyl-prolyl isomerase Pin1 was obtained by PCR amplification using the ATCC 555784 as template. ATCC 555784 contained cDNA encoding the Pin1 cloned into the pT7T3D-pac (http://www.atcc.org). The primers used to PCR amplify PIN1 gene were 5'GGA TCC ATG GCG GAC GAG GAG AAG CTG CCG CCC-3' and 5'GGA TCC TCA CTC AGT GCG GAG GAT GAT GTG GAT GCC-3'. The PCR product was cloned into pCR2.1 TOPO (Invitrogen, Carlsband, CA) to generate plasmid pBG3301. This plasmid was digested with BamH1 and the fragment corresponding to the Pinl was cloned into the BamH1 site of pBG1380, a derivative of pBHR1 to generate plasmid pBG3302. The plasmid pBHR1 is a cloning vector with broad hostrange maintenance and it stably replicates in all gram-negative organisms under standard growth conditions and the appropriate selective pressure [27]. The plasmid pBG1380 has been modified to express the protein of interest under the control of the nifH promoter, which is a strong 


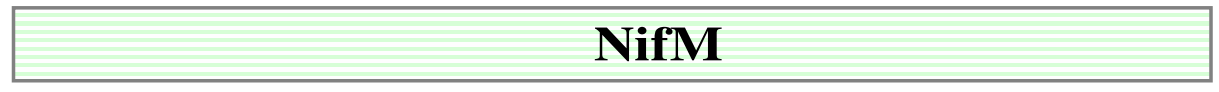

\section{Pin1}

1

1
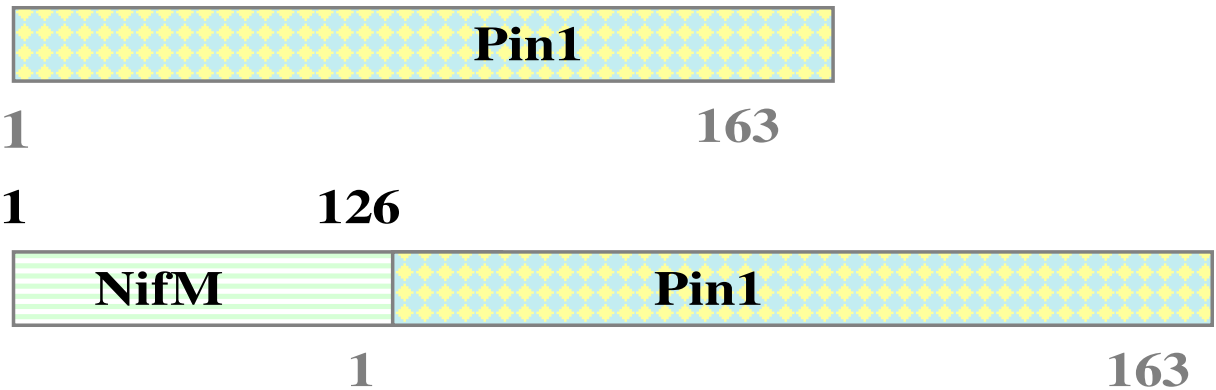

Fig.3: The physical map of various proteins used in genetic complementation. The NifM protein is 292 amino acid long containing both the amino terminal interaction domain and the carboxyl terminal PPIase domain. The Pin1 protein is 163 amino acid long which is homologous to the carboxyl terminus of the NifM protein was unable to complement the function of NifM due to the absence of the probable interaction domain. The construction of the 289 amino acid NifM/Pin1 chimera is described in the 'materials and methods' and this chimera was able to complement the function of NifM.

promoter and utilizes the $A$. vinelandii transcriptional and translational regulation to over produce specific target protein; it also contains the chloramphenicol resistance gene as a marker for selection in $A$. vinelandii and E.coli strains. This vector also contains unique EcoRV, BamHI and HinDIII cloning sites downstream of the nifH promoter which allows the use of these sites for cloning of specific gene constructs into pBG1380 and express the proteins of interest in $A$. vinelandii under nitrogen fixing conditions. The plasmid pBG3302 was used to express the Pin1 protein in A. vinelandii BG98 and investigate whether the PPIase activity of the Pin1 protein can help in the maturation of the Fe protein and lead to the $\mathrm{Nif}^{+}$phenotype of $A$. vinelandii BG98. NifM is a PPIase [14]. NifM is required for the activation and stabilization of the NifH of nitrogenase [9]. The plasmid pBG3302 was transformed into $A$. vinelandii $\mathrm{BG} 98$ and the strain expressing the Pin1 protein was designated as A. vinelandii BG3302 (Table 1 and Fig. 3). The A. vinelandii BG3302 over expresses the Pin1 protein under nitrogen limiting conditions as it is under the control of the nifH promoter, and if the peptidyl prolyl isomerase activity of the Pin1 protein is sufficient to take up the function of the NifM protein then it should lead to the activation and stabilization of the NifH of the nitrogenase leading to a $\mathrm{Nif}^{+}$phenotype of the strain. It was observed that the A.vinelandii BG3302 did not show any growth on either Burkes Nitrogen free plates (Fig. 4) or in Burkes Nitrogen free liquid medium (Fig. 5). This indicated that the peptidyl prolyl isomerase activity of the human Pin1 protein alone is not sufficient to take up the function of the NifM protein in A. vinelandii. 


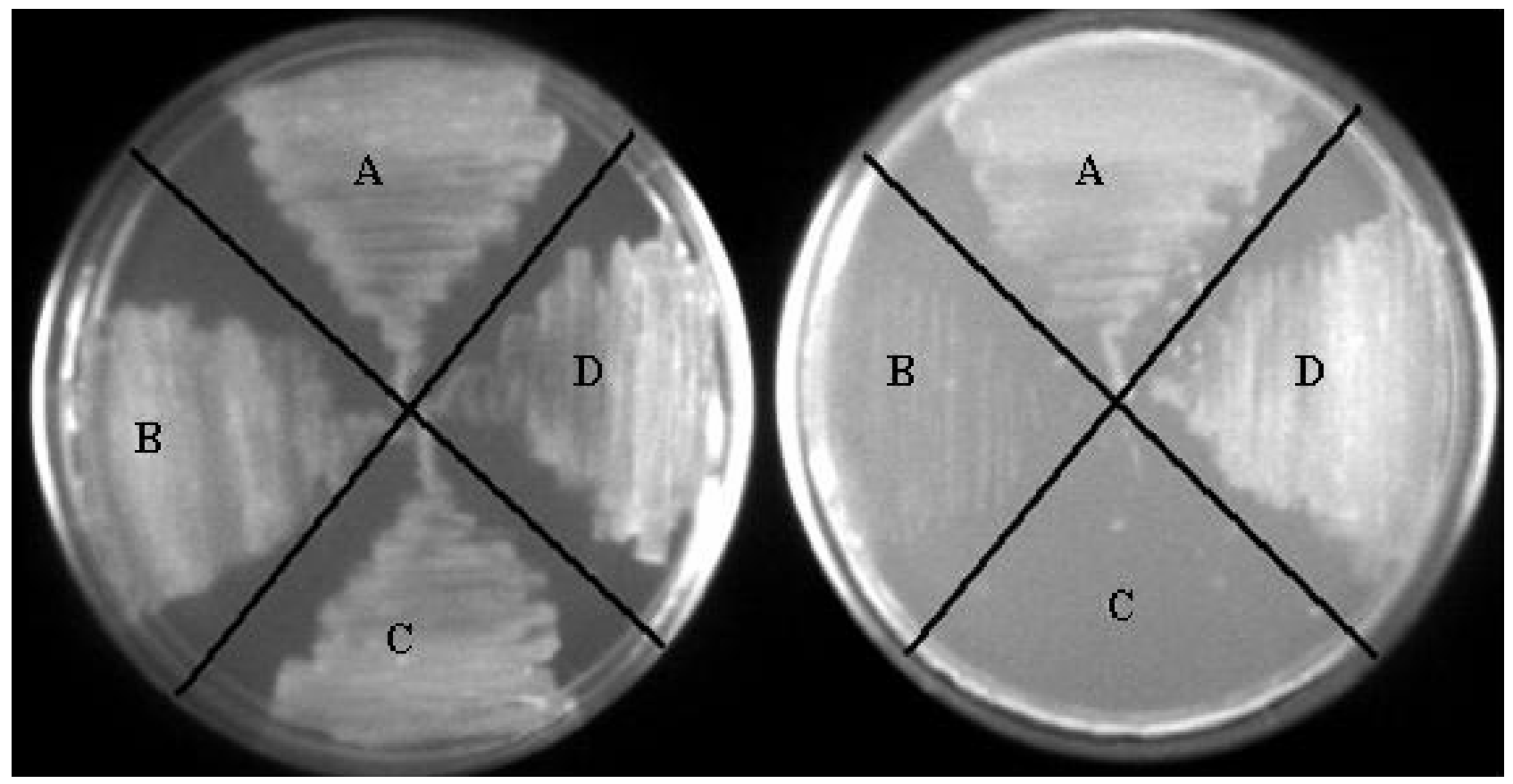

Fig. 4: Growth characteristics of A.vinelandii strains in $\mathrm{BN}^{+}$and $\mathrm{BN}^{-}$agar plates. The growth properties of various strains when cultured on $\mathrm{BN}^{+}$agar (left panel) or on $\mathrm{BN}^{-}$agar (right panel). The strains streaked on the plates are A.vinelandii OP (A), A.vinelandii BG98 (B), A.vinelandii BG3302 (C), A.vinelandii BG3305 (D)

\section{The NifM/Pin1 chimera complements the NifM function in $A$. vinelandii:}

Since the Pin1 protein could not complement the function of the NifM protein we hypothesized that the amino terminus of the NifM may be required for its recognition of the Fe protein and in its absence the Pin1 protein is unable to interact with the $\mathrm{NifH}$ and therefore unable to complement the function of NifM. To verify our hypothesis that the amino terminus of the NifM may be required for the recognition of the NifH, a 378 bp DNA fragment encoding the 126 amino acids of the amino terminus of the NifM was obtained by PCR amplification using $A$. vinelandii $\mathrm{OP}$ chromosome as template. The primers used to amplify the region encoding amino terminal 126 amino acid of NifM were 5'GAT ATC ATG GCA TCT GAG CGT CTC GCC GAC GGC GAC AGC-3' and 5'GAT ATC GGT GTC GCT GAT CTC CGG CAG GCC GGC GCA GAC3'. This fragment was cloned into pCR2.1 TOPO to generate plasmid pBG3304. This plasmid was digested with $E c o \mathrm{RV}$ and the fragment was cloned into EcoRV digested pBG3302 to generate plasmid pBG3305. The strain carrying this plasmid expresses a translational fusion of the amino terminal 126 amino acids of the NifM and the Pin1 protein (Fig. 3). This plasmid was used to express the amino-terminal NifM-Pin1 chimera in A. vinelandii and analyze whether the PPIase activity of the Pin1 protein in combination with the amino terminal 126 amino acids of NifM can take up the function of NifM and lead to the maturation and activation of the Fe protein and confer the $\mathrm{Nif}^{+}$phenotype to A. vinelandii BG98 (Fig. 3). The plasmid pBG3305 was introduced into A. vinelandii $\mathrm{BG} 98$ and the strain expressing the amino terminus of the NifM and the Pin1 protein was designated as $A$. vinelandii BG3305. This strain showed growth on both Burkes Nitrogen free plates (Fig. 4) as well as in the Burkes Nitrogen free liquid medium (Fig. 5). While the wild type showed the highest growth rate, the growth of $A$. vinelandii BG3305 was comparatively lower but it was much higher than that of the A. vinelandii BG98 as well as A. vinelandii $\mathrm{BG} 3302$. This indicates that the peptidyl prolyl isomerase activity of the Pin1 protein combined with the amino terminus of the NifM is sufficient to take up the function of the NifM protein in the NifM mutant strain A. vinelandii BG98. These results imply that the amino terminus of the NifM may act as the recognition domain and the carboxyl terminus acts as the PPIase domain and both this domains are required for the activity of NifM. These results are consistent with the findings that specific binding modules fused to PPIase catalytic domain define many of the critical functions of essential parvulins. 


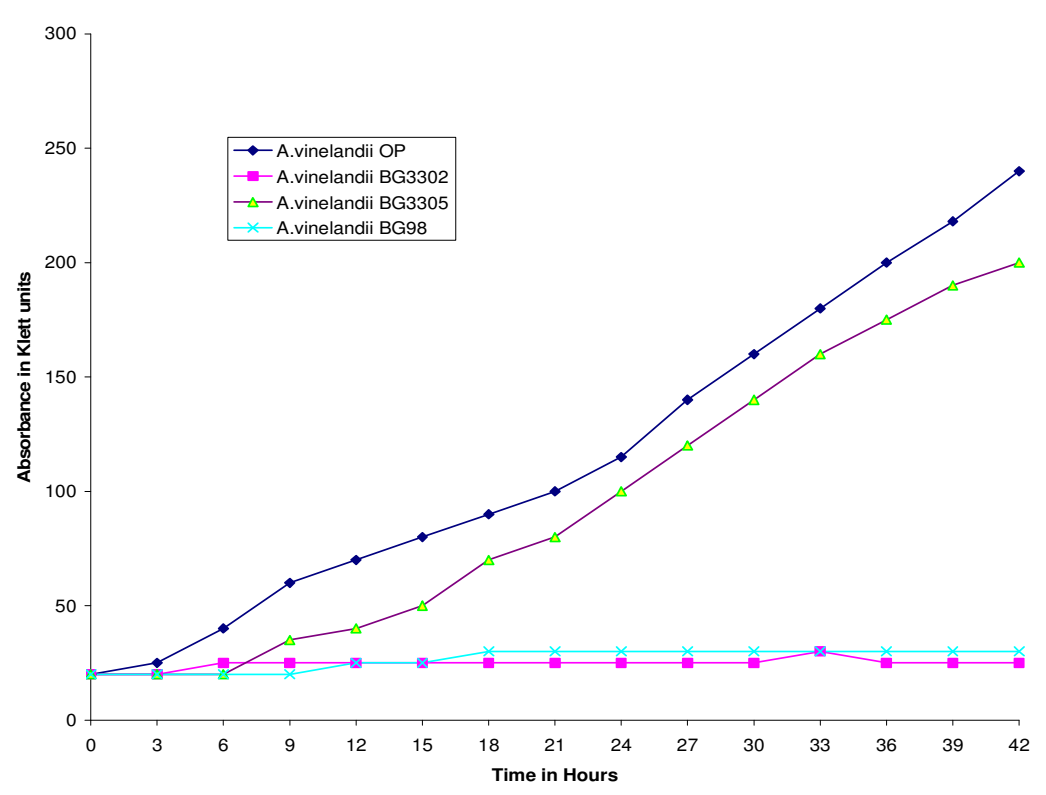

Fig.5: Growth characteristics of A. vinelandii strains in $\mathrm{BN}^{-}$liquid medium. Overnight cultures were prepared by inoculating freshly growing colonies from agar plate into Burke's medium supplemented with ammonium acetate. Cells from the overnight culture were collected and washed with $\mathrm{BN}^{-}$and then transferred to side-arm flasks that contained sterile $\mathrm{BN}^{-}$.Cell densities were recorded over 42 hour time periods. This analysis showed that the $A$. vinelandii $\mathrm{BG} 3305$ strain showed slower growth compared to $A$. vinelandii O.P and the strains $A$. vinelandii $\mathrm{BG} 98$, A. vinelandii BG3302 did not show any growth.

\section{ACKNOWLEDGMENTS}

Part of this work was done at Bowling Green State University, Ohio. This research is supported by NSF to NG and LP. We thank members of Gavini/Pulakat laboratory for helpful discussions and technical help.

\section{REFERENCES}

1. Burgess, B.K. and D.J. Lowe. 1996. Mechanism of molybdenum nitrogenase. Chem. Rev., 96:2983-3011.

2. Rubio, L.M. and P.W. Ludden. 2005. Maturation of nitrogenase: a biochemical puzzle. J. Bacteriol., 187:405-414.

3. Christiansen, J., D.R. Dean and L.C. Seefeldt. 2001. Mechanistic features of the Mo-containing nitrogenase. Annu. Rev. Plant. Physiol. Plant. Mol. Biol., 52:269-295.

4. Howard, J.B. and D.C. Rees. 1996. Structural basis of biological nitrogen fixation. Chem. Rev., 96:2965-2982.

5. Georgiadis, M.M., H. Komiya, P. Chakrabarti, D. Woo, J.J. Kornuc and D.C. Rees. 1992. Crystallographic structure of the nitrogenase Iron protein from Azotobacter vinelandii. Science, 257:1653-1659.
6. Jacobson, M.R., K.E. Brigle, L.T. Bennett, R.A. Setterquist, M.S. Wilson, V.L.Cash, J.Beynon, W.E. Newton and D.R.Dean. 1989. Physical and genetic map of the major nif gene cluster from Azotobacter vinelandii. J. Bacteriol., 171:10171027.

7. Roberts, G.P, T. MacNeil, D. MacNeil and WJ Brill. 1978. Regulation and characterization of protein products coded by the nif (nitrogen fixation) genes of Klebsiella pneumoniae. J. Bacteriol. 136:267-79.

8. Paul, W. and M. Merrick. 1989. The roles of the nifW, nifZ and nifM genes of Klebsiella pneumoniae in nitrogenase biosynthesis. Eur. J. Biochem., 178:675-682.

9. Howard, K.S., P.A. McLean, F.B. Hansen, P.V. Lemley, K.S. Koblan and W.H. Ormejohnson. 1986. Klebsiella pneumoniae nifM gene product is required for stabilization and activation of nitrogenase iron protein in Escherichia coli. J. Biol. Chem., 261:772-778.

10. Petrova, N., L. Gigova and P. Venkov. 2002. Dimerization of Rhizobium meliloti NifH protein in Saccharomyces cerevisiae cells requires simultaneous expression of NifM protein. Int. J. Biochem. Cell Biol., 34:33-42. 
11. Zou, L., M.C. Baguinon, X.L. Gu, S.Y. Gu, Y. $\mathrm{Yu}$ and L.C. Davis. 2000. Interaction with magnesium and ADP stabilizes both components of nitrogenase from Klebsiella pneumoniae against urea denaturation. Protein Sci., 9:121-128.

12. Jacobson, M.R., V.L. Cash, M.C. Weiss, N.F. Laird, W.E. Newton and D.R. Dean. 1989. Biochemical and genetic analysis of the nifUSVWZM cluster from Azotobacter vinelandii. Mol. Gen. Genet., 219:49-57.

13. Fu, W.G., R.F. Jack, T.V. Morgan, D.R. Dean and M.K. Johnson. 1994. NifU gene product from Azotobacter vinelandii is a homodimer that contains 2 identical [2Fe-2S] clusters. Biochemistry, 33:13455-13463.

14. Gavini, N. and L. Pulakat. 2002. Role of NifM in maturation of the Fe-protein of Nitrogenase, p. 228-232, In, Nitrogen Fixation- Global Perspectives eds. T. M. Finan, M.R. O'Brian, D.B. Layzell, J.K. Vessey and W.Newton. CABI Publishing, New York.

15. Fischer, G. 1994. Peptidyl-prolyl cis/trans isomerases and their effectors. Angew. Chem. Int. Ed. Engl., 33:1415-1436.

16. Fischer G, H.Bang and C. Mech. 1984. Determination of enzymatic catalysis for the cistrans isomerization of peptide binding in proline containing peptides. Biomed. Biochim. Acta., 43:1101-11.

17. Yaffe, M.B., M. Schutkowski, M.H. Shen, X.Z. Zhou, P.T. Stukenberg, J.U. Rahfeld, J. Xu, J. Kuang, M.W. Kirschner, G. Fischer, L.C. Cantley and K.P. Lu. 1997. Sequence-specific and phosphorylation-dependent proline isomerization: A potential mitotic regulatory mechanism. Science, 278:1957-1960.

18. Lu, K.P., Y.C. Liou and X.Z. Zhou. 2002. Pinning down proline-directed phosphorylation signaling. Trends Cell Biol., 12:164-172.

19. Zhou, X.Z., P.J. Lu, G. Wulf and K.P. Lu. 1999. Phosphorylation-dependent prolyl isomerization: a novel signaling regulatory mechanism. Cell. Mol. Life Sci.,56:788-806.

20. Sudol, M. 1996. Structure and function of the WW domain. Prog. Biophys. Mol. Biol., 65:113132.
21 .Lu,P.J.,X.Z.Zhou, M.Shen and K.P.Lu. 1999. Function of WW domains as phosphoserine- or phosphothreonine-binding modules. Science, 283:1325-8

22. Rahfeld, J.U., A. Schierhorn, K. Mann and G. Fischer. 1994. A novel peptidyl-prolyl Cis/trans isomerase from Escherichia coli. FEBS Lett. 343:65-69.

23. Lei, S., L. Pulakat and N. Gavini. 1999. Regulated expression of the nifM of Azotobacter vinelandii in response to molybdenum and vanadium supplements in Burk's nitrogen free growth medium. Biochem. Biophys. Res. Commun., 264:186-190.

24. Strandberg G.W and P.W.Wilson. 1968. Formation of the nitrogen-fixing enzyme system in Azotobacter vinelandii. Can. J. Microbiol., 14:25-31.

25. Sambrook, J., E.F. Fritsch and T. Maniatis. 1992. Molecular Cloning: A Laboratory Manual. Cold Spring Harbor Laboratory, Cold Spring Harbor, New York.

26. Suh, M.H., L. Pulakat and N. Gavini. 2003. Functional expression of a fusion-dimeric $\mathrm{MoFe}$ protein of nitrogenase in Azotobacter vinelandii. J. Biol. Chem., 278:5353-5360.

27. Szpirer, C.Y., M. Faelen and M. Couturier. 2001. Mobilization function of the pBHR1 plasmid, a derivative of the broad-host-range plasmid pBBR1. J. Bacteriol., 183:2101-2110.

28. Ranganathan, R., K.P. Lu, T.Hunter and J.P Noel.1997. Structural and functional analysis of the mitotic rotamase Pin1 suggests substrate recognition is phosphorylation dependent. Cell, 89(6): 875-86

29. Thompson J.D., Higgins D.G. and Gibson T.J. 1994.CLUSTAL W: improving the sensitivity of progressive multiple sequence alignment through sequence weighting, position-specific gap penalties and weight matrix choice. Nucleic Acids Res. 22:4673-4680

30. Schwede, T., J. Kopp, N.Guex and M.C Peitsch.2003. SWISS-MODEL: An automated protein homology-modeling server. Nucleic Acids Res. 31(13):3381-5. 